\title{
Optimising RAPD-PCR for Screening the Link of RAPD Markers to an Acid-resistant Gene in Oenococcus oeni
}

\author{
S. Liu ${ }^{1}, \mathrm{~L} . \mathrm{He}^{2}, \mathrm{X} \cdot \mathrm{Li}^{1}, \mathrm{X} \cdot \mathrm{Li}^{3 *}$ \\ (1) College of Enology, Northwest A \& F University, Yangling, Shannxi Province, 712100, China \\ (2) College of Horticulture, Northwest A \& F University, Yangling, Shannxi Province, 712100, China \\ (3) Western Institute for Food Safety and Security, University of California, Davis, California, 95618, USA
}

Submitted for publication: June 2011

Accepted for publication: September 2011

Key words: Oenococcus oeni, acid resistant, RAPD markers, gene

\begin{abstract}
RAPD-PCR conditions were optimised for screening RAPD markers linked to the acid-resistant gene in Oenococcus oeni. Two (S40, S333) out of 45 random primers were capable of producing stable polymorphism in $O$. oen $i$ isolates. Thirty-three acid-resistant isolates and nine acid-sensitive isolates of $O$. oeni were used for screening RAPD markers linked to the acid-resistant gene. Specific bands of S40-1400 and S333-650 were amplified in $31(94 \%)$ and $33(100 \%)$ of 33 acid-resistant $O$. oeni isolates. The optimised RAPD-PCR method can potentially be used for the fast screening of acid-resistant $O$. oen $i$ strains.
\end{abstract}

\section{INTRODUCTION}

Alcoholic fermentation by yeasts and the transformation of malic acid into lactic acid by lactic acid bacteria are two major steps in winemaking. Many winemakers favour malolactic fermentation because it improves the organoleptic properties of wines (Sancho et al., 1998). Among the many lactic acid bacteria species present in grapes, Oenococcus oeni is one of the species that triggers malolactic fermentation. Although many strains of $O$. oen $i$ are well adapted to such a harsh ecological medium as wine, some strains do not grow in wine due to the presence of various compounds not favoured by the organisms (Augagneur et al., 2007). Environmental factors, such as low $\mathrm{pH}$, high concentrations of alcohol and $\mathrm{SO}_{2}$, and a lack of nutrients, also inhibit the growth of some strains of O. oeni (Nehme et al., 2008). In this context, selecting and using acid-resistant strains of $O$. oeni are increasingly being recognised as critical for successful malolactic fermentation (Li et al., 2006).

A traditional method for selecting acid-resistant $O$. oen $i$ strains is to evaluate the ability of bacteria to adapt to various factors present in wine $\left(\mathrm{pH}\right.$, alcohol, $\mathrm{SO}_{2}$ etc.) (Carreté et al., 2005). However, the wine industry needs various single-factor tests and multiple-factor tests to evaluate and select strains of interest. In addition, $O$. oeni usually grows slowly and requires a nutrient-rich medium and anaerobic environment. The traditional method does not meet the current needs of the industry because of its inefficiency and inaccuracy. Molecular-based methods have been approved as rapid and reliable tools for the screening of $O$. oeni strains (de Los Reyes-Gavilán et al., 1992). Very recently, a multiplex PCR method has been developed for identifying and typing
O. oeni (Araque et al., 2009). With a broad aim of selecting acid-resistant strains of $O$. oeni, the objective of the present study was to optimise the RAPD-PCR, to characterise the acid-resistant strains and acid-sensitive strains of $O$. oeni at the DNA level, and to detect RAPD specific molecular markers linked to acid-resistant genes in $O$. oeni.

\section{MATERIALS AND METHODS}

Forty-two $O$. oeni isolates from the culture collection at the Microbiology Laboratory of the College of Enology, Northwest A \& F University, China were used for this work. All these isolates were isolated during the natural fermentation process in wineries. In terms of the locations of the wineries, the identities of these isolates were initialised with 'NX', 'SD' and 'SX' respectively and followed by batch numbers. NX represents isolates from Guangxia Winery in NingXia Autonomous Region; SD represents isolates from Yantai Winery in ShanDong Province; and SX represent isolates from Yangling Winery in ShannXi Province, China. The susceptibilities of $O$. oeni isolates to acid (acid resistant or acid sensitive) were determined by the growth of bacteria in acidic culture. Briefly, each isolate was cultured in acidic tomato broth (ATB) $(\mathrm{pH} \mathrm{4.8)}$ and log phase bacteria were inoculated into the ATB ( $\mathrm{pH} 3.2)$ at concentrations of $5 \times 10^{7}$ colony forming units (CFU)/mL. After incubation at $26^{\circ} \mathrm{C}$ for four days, the optical densities (OD) of the bacteria solutions were measured three times using a spectrophotometer at $600 \mathrm{~nm}$. Isolates that yielded mean OD values $\geq 0.600$ were considered as acid-resistant strains, while those with mean OD values $\leq 0.300$ were considered to be acid-sensitive

*Corresponding author: xdli@ucdavis.edu

Aknowledgements: This collaborative research between the College of Enology, Northwest A\&F University and the Western Institute for Food Safety and Security, University of California Davis was supported by China's earmarked fund for modern agro-industry technology research system (NYCYTX-30) 
strains. We have determined that the method of using the OD value is closely correlated with the method of direct plating and counting CFU $(\mathrm{R}>0.90)$ (data not shown) in determining the ability of $O$. oeni to grow at low $\mathrm{pH}$. Among the 42 isolates, nine (NX-2g, NX-4f, NX-3c, NX-4a, NX$4 \mathrm{~d}, \mathrm{NX}-2 \mathrm{~h}, \mathrm{NX}-3 \mathrm{e}, \mathrm{NX}-3 \mathrm{~d}$, and NX-3h) were determined as being acid-sensitive strains and the 33 other isolates as being acid-resistant strains. The ability of acid-resistant strains to grow at $\mathrm{pH}<3.2$ was assessed by culture bacteria under the same conditions as above, but at $\mathrm{pH}$ 3.0. After four days of growth, the OD values of all the strains were $<0.200$, except for that of strain SD-2a, which was $>0.200$ but $<0.300$.

Genomic DNA was extracted from $O$. oeni isolates using a phenol/chloroform extraction method previously described by Doulis et al. (1999). Genomic DNA from the isolate SD-2a was used as template DNA and a random primer S333 was used as default primer for optimising the RAPD-PCR. Factors evaluated for RAPD-PCR optimisation included doses of Taq DNA polymerase, concentrations of dNTP, primer, $\mathrm{Mg}^{2+}$ and template DNA, and temperature for annealing. Single-factor tests were conducted to determine the best conditions for each factor. The RAPD-PCR reaction system was optimised through a combination of various factors as described by Schauder et al. (2001). The gradients of factors evaluated for RAPD-PCR optimisation were 0.5 , 1.0, 1.5 and 2.0 units of Taq DNA polymerase; 80, 120, 160, 180 and $200 \mu \mathrm{mol} / \mathrm{L}$ of dNTP; 0.2, 0.4, 0.6 and $0.8 \mu \mathrm{mol} / \mathrm{L}$ of primer; $0.5,1.5,2.0,2.5$ and $3.0 \mathrm{mmol} / \mathrm{L}$ of $\mathrm{Mg}^{2+} ; 5,10,20$, 40 and $60 \mathrm{ng} / \mathrm{L}$ of DNA template; and $34,36,38$ and $40^{\circ} \mathrm{C}$ of annealing temperature. PCR was conducted by annealing at $94^{\circ} \mathrm{C}$ for $5 \mathrm{~min}$, followed by 45 cycles, each with $1 \mathrm{~min}$ at $94^{\circ} \mathrm{C}, 1 \mathrm{~min}$ at $36^{\circ} \mathrm{C}$ and $1.5 \mathrm{~min}$ at $72^{\circ} \mathrm{C}$, and a final extension at $72{ }^{\circ} \mathrm{C}$ for $5 \mathrm{~min}$. PCR products were examined using $2 \%$ agarose gel electrophoresis stained with ethidium bromide. The optimised conditions for each factor were achieved by examining the presence and specificity of bands in agarose gels.
Forty-five randomly selected primers (Table 1) previously used for screening O. oeni in China (Zhang, 2001) were synthesised by Generay Biotech Co. LTD (Shanghai, China). Primers were primarily screened with the optimised RAPD-PCR using DNA template from isolate SD-2a, and those that produced positive products were further selected in an allele pool, using the method described by Kilstrup et al. (1997). This two-step procedure for selecting primers was capable of characterising the genomic differences between bacteria isolates (Ingmer et al., 1999).

\section{RESULTS AND DISCUSSION}

According to the outcomes of repeated experiments for each factor examined, the optimised primary conditions were 1.0 $\mathrm{U}$ Taq polymerase, $160 \mu \mathrm{mol} / \mathrm{L} \mathrm{dNTP,} 0.4 \mu \mathrm{mol} / \mathrm{L}$ random primer, $3.0 \mathrm{mmol} / \mathrm{L} \mathrm{Mg}^{2+}$ and $10 \mathrm{ng}$ DNA template in a $25 \mu \mathrm{L}$ reaction system. Electrophoresis of comparisons of the gradients of each factor is shown in Fig. 1. Annealing temperature was optimised at $36^{\circ} \mathrm{C}$ and the PCR regimen was programmed as $94^{\circ} \mathrm{C}$ for $5 \mathrm{~min}$, followed by 45 cycles of $1 \mathrm{~min}$ at $94^{\circ} \mathrm{C}, 1 \mathrm{~min}$ at $36^{\circ} \mathrm{C}$ and $1.5 \mathrm{~min}$ at $72^{\circ} \mathrm{C}$, and a final extension at $72^{\circ} \mathrm{C}$ for $5 \mathrm{~min}$.

Among the 45 random primers tested, 40 (88.8\%) yielded positive amplification of PCR products. The 40 primers were further tested on the allele pool and 36 primers produced stable and repeatable results. Of the 36 primers, six produced primer-specific bands in electrophoresis. The six primers were S25, S32, S33, S40, S178 and S333, which produced specific band sizes of 700, 600-700, $>1500,1400,900$ and $650 \mathrm{bp}$ respectively. Using the six primers that yielded primer-specific amplifications, the DNA polymorphisms present in the gene pool were further verified in a single isolate of $O$. oeni. Results from repeated experiments revealed that only two of the six primers, S40 and S333, exhibited a stable polymorphism in single isolates. With the primer S40, genomic DNA of 31 (94\%) of the 33 acid-resistant isolates amplified a clear and stable band of

TABLE 1

The random primers screened for RAPD-PCR.

\begin{tabular}{lccccc}
\hline Primer & Sequence & Primer & Sequence & Primer & Sequence \\
\hline S1 & GTTTCGCTCC & S16 & TTTGCCCGGA & S31 & CAATCGCCGT \\
S2 & TGATCCCTGG & S17 & AGGGAACGAG & S32 & TCGGCGATAG \\
S3 & CATCCCCCTG & S18 & CCACAGCAGT & S33 & CAGCACCCAC \\
S4 & GGACTGGAGT & S19 & ACCCCCGAAG & S34 & TCTGTGCTGG \\
S5 & TGCGCCCTTC & S20 & GGACCCTTAC & S35 & TTCCGAACCC \\
S6 & TGCTCTGCCC & S21 & CAGGCCCTTC & S36 & AGCCAGCGAA \\
S7 & GGTGACGCAG & S22 & TGCCGAGCTG & S37 & GACCGCTTGT \\
S8 & GTCCACACGG & S23 & AGTCAGCCAC & S38 & AGGTGACCGT \\
S9 & TGGGGGACTC & S24 & AATCGGGCTG & S39 & CAAACGTCGG \\
S10 & CTGCTGGGAC & S25 & AGGGGTCTTG & S40 & GTTGCGATCC \\
S11 & GTAGACCCGT & S26 & GGTCCCTGAC & S90 & AGGGCCGTCT \\
S12 & CCTTGACGCA & S27 & GAAACGGGTG & S178 & TGCCCAGCCT \\
S13 & TTCCCCCGCT & S28 & GTGACGTAGG & S288 & AGGCAGAGCA \\
S14 & TCCGCTCTGG & S29 & GGGTAACGCC & S333 & GACTAAGCCC \\
S15 & GGAGGGTGTT & S30 & GTGATCGCAG & S412 & GGGACGTTGG \\
\hline
\end{tabular}




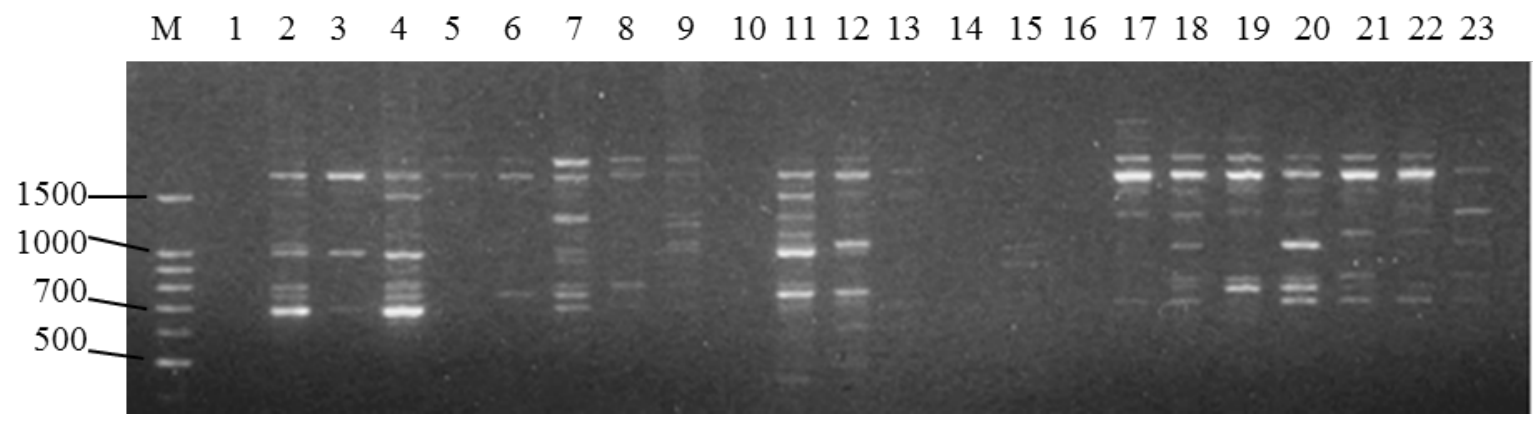

FIGURE 1

RAPD profiles obtained from $O$. oeni using different concentrations of five primary factors. Lanes $1-4$ represent $0.5,1.0$, 1.5, 2.0 UTaq DNA polymerase; 5 - 9 represent 80, 120, 160, 180, $200 \mu \mathrm{mol} / \mathrm{L}$ dNTP; 10 - 13 represent 0.2, 0.4, 0.6, 0.8 $\mu \mathrm{mol} / \mathrm{L}$ of primer; 14 - 18 represent $0.5,1.5,2.0,2.5,3.0 \mathrm{mmol} / \mathrm{L}$ of $\mathrm{Mg}^{2+} ; 19-23$ represent 5, 10, 20, 40, 60 ng of genomic DNA, respectively. M: Molecular marker.
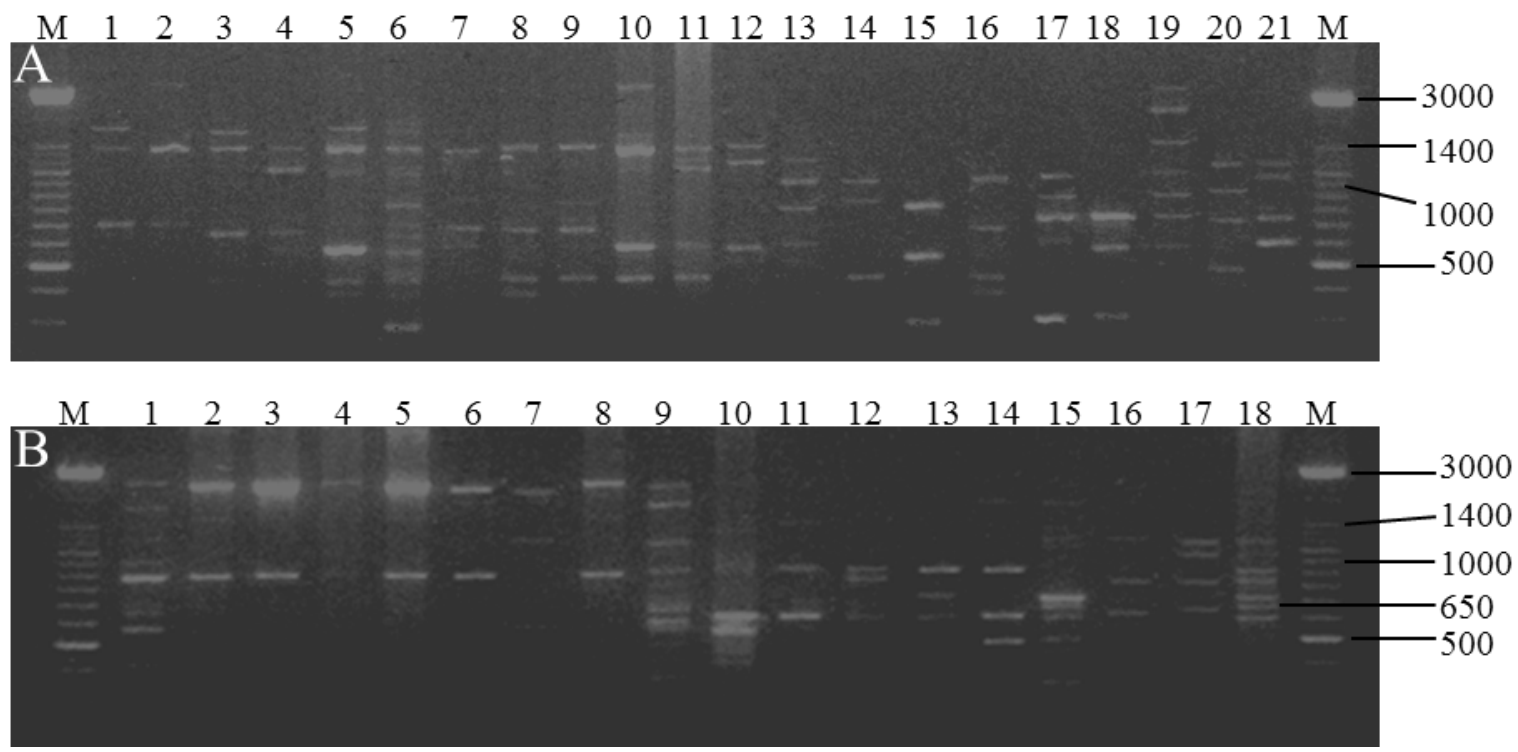

FIGURE 2

A. Genomic DNA amplification in single $O$. oeni isolates using primer S40. Lanes 1 - 12 represent acid-resistant isolates: SX-1a, SD-1f, SD-2hg, SD-2ji, SD-2a, NX-3g, NX-2f, NX-3f, NX-1g, NX-1h, NX-2b, SD-1b; Lanes 13 - 21 represent acidsensitive isolates: NX-2g, NX-4f, NX-3c, NX-4a, NX-4d, NX-2h, NX-3e, NX-3d, NX-3h. M: Molecular marker. A band of $1400 \mathrm{bp}$ was amplified in the acid-resistant isolates but not in the acid-sensitive isolates, except for NX-3e. B. Genomic DNA amplification in single $O$. oeni isolates using primer S333. Lanes 1 - 9 represent acid-sensitive isolates: NX-2g, NX-4f, NX3c, NX-4a, NX-4d, NX-2h, NX-3e, NX-3d, NX-3h; Lanes 10 - 18 represent acid-resistance isolates: SX-1a, SD-1f, SD-2hg, SD-2ji, SD-1b, SD-2a, NX-3g, NX-2f, NX-3f; M: Molecular marker. A band of 650 bp was amplified in the acid-resistant isolates, but not in the acid-sensitive isolates, except for isolate NX-3h.

$1400 \mathrm{bp}$. In contrast, of the nine acid-sensitive isolates, eight $(88.9 \%)$ did not exhibit the specific band of $1400 \mathrm{bp}$. Figure $2 \mathrm{~A}$ shows the comparison of representative acid-resistant isolates and acid-sensitive isolates amplification with primer S40, from which we can see that all acid-resistant isolates exhibit the band $1400 \mathrm{bp}$, but that all acid-sensitive isolates except for NX-3e did not exhibit the band $1400 \mathrm{bp}$. With the primer S333, a band of 650 bp was present in $33(100 \%)$ of the 33 acid-resistant $O$. oeni isolates, but not in eight (88.9\%) of the nine acid-sensitive isolates (except for isolate NX-3h) (Fig. 2B).
The presence and specificity of bands derived from the two primers indicate that the specific bands of S40-1400 and S333-650 are molecular markers linked to the gene of acid resistance in the 42 isolates of $O$. oeni screened. The markers of the two specific bands linked to the gene for acid resistance in $O$. oeni isolates were achieved through selection from 45 random primers and confirmation in a single $O$. oeni isolate. No reports could be found on established acid-resistant $O$. oeni strains and the relevant technological properties of such strains. However, the linkage between tolerance to stress and technological properties has been observed 
in $O$. oeni strains. For example, in one study, two types of $O$. oeni strains with different physiological characteristics relevant to environmental stress were distinguished using PCR and electrophoresis (Renouf et al., 2009). In another study, positive correlations between malolactic activity and the ability to develop and tolerate stress conditions in selected $O$. oeni strains were observed using RAPD-PCR (Capozzi et al., 2010). Nevertheless, the linkage between physiological characteristics and tolerance to stress in $O$. oeni has not been confirmed by researchers nor widely accepted by the wine industry. One objective of the present trial was to establish a method for fast screening acidresistant $O$. oeni strains. Although preliminary data from this work was promising, future studies are definitively needed to further characterise the $O$. oeni strains and to assess the methodology by determining the genotypes of $O$. oeni strains and sequencing the PCR products derived from each primer used.

\section{CONCLUSIONS}

In summary, the findings of this study demonstrate the presence of DNA polymorphism in genomic DNA between acid-resistant and acid-sensitive $O$. oeni strains. The PCR conditions were optimised and the RAPD-PCR reaction system was established and found to be a suitable application in our laboratories. We have been using this technique in our laboratories for characterising $O$. oen $i$ isolates and there is the potential of its application in the winemaking industry for the fast screening of acid-resistant $O$. oeni strains.

\section{LITERATURE CITED}

Araque, M.I., Bordons, A. \& Reguant, C., 2009. A multiplex PCR method for simultaneous species identification and strain typification of Oenococcus oeni. World J. Microbiol. Biotechnol. 25(1), 15-18.

Augagneur, Y., Ritt, J.F., Linares, D.M., Remize, F., Tourdot-Maréchal, R., Garmyn, D. \& Guzzo, J., 2007. Dual effect of organic acids as a function of external pH in Oenococcus oeni. Arch. Microbiol. 188(2), 147-157.

Capozzi, V., Russo, P., Beneduce, L., Weidmann, S., Grieco F., Guzzo, J. \& Spano, G., 2010. Technological properties of Oenococcus oeni strains isolated from typical southern Italian wines. Lett. Appl. Microbiol. 50(3), 327-334.
Carreté, R., Reguant, C., Bordons, A. \& Constantí, M., 2005. Relationship between a stress membrane protein of Oenococcus oeni and glyceraldehyde3-phosphate dehydrogenases. Appl. Biochem. Biotechnol. 127(1), 43-51.

De Los Reyes-Gavilán, C.G., Limsowtin, G.K., Tailliez, P., Séchaud, L. \& Accolas, J.P., 1992. A Lactobacillus helveticus-specific DNA probe detects restriction fragment length polymorphisms in this species. Appl. Environ. Microbiol. 58(10), 3429-3432.

Doulis, A.G., Harfouche, A.L. \& Aravanopoulos, F.A., 1999. Rapid, high quality DNA isolation from Cypress (Cupressus sempervirens L.) needles and optimization of the RAPD marker technique. Plant Mol. Biol. Rep. 17(4), 411-412.

Ingmer, H., Vogensen, F.K., Hammer, K. \& Kilstrup, M., 1999. Disruption and analysis of the clpB, cipC, and cipE genes in Lactococcus lactis: ClpE, a new CIp family in gram-positive bacteria. J. Bacteriol. 181(7), 2075-2083.

Kilstrup, M., Jacobsen, S., Hammer, K. \& Vogensen, F.K., 1997. Induction of heat shock proteins Dnak, GroEL, and GroES by salt stress in Lactococcus lactis. Appl. Environ. Microbiol. 63(5), 1826-1837.

Li, H., Zhang, C. \& Liu, Y., 2006. Species attribution and distinguishing strains of Oenococcus oeni isolated from Chinese wines. World J. Microbiol. Biotechnol. 22(5), 515-518.

Nehme, N., Mathieu, F. \& Taillandier, P., 2008. Quantitative study of interactions between Saccharomyces cerevisiae and Oenococcus oeni strains. J. Ind. Microbiol. Biotechnol. 35(7), 685-693.

Renouf, V., Vayssieres, L.C., Claisse, O. \& Lonvaud-Funel, A., 2009. Genetic and phenotypic evidence for two groups of Oenococcus oeni strains and their prevalence during winemaking. Appl. Microbiol. Biotechnol. 83(1), 85-97.

Sancho, J., García, C.E.G. \& Carbó, R., 1998. Influencia de la graduación alcohólica en la fermentación maloláctica. Alimentación Eequipos y tecnología 17(2), 71-74.

Schauder, S., Shokat, K., Surette, M.G. \& Bassler, B.L., 2001. The LuxS family of bacteria autoinducers: biosynthesis of a novel quorum-sensing signal transduction molecule. Mol. Microbiol. 41(2), 463-476.

Zhang, C.H., 2001. Screening and isolation of Oenococcus oeni and malic-lactic acid fermentation. Ph.D. dissertation, College of Enology, Northwestern A \& F University, China. 